\begin{tabular}{|c|c|}
\hline Title & $\begin{array}{l}\text { Molecular dynamics simulations of the nucleation of water: Determining the sticking probability and formation energy } \\
\text { of acluster }\end{array}$ \\
\hline Author(s) & Tanaka, Kyoko K.; Kawano, A kio; Tanaka, Hidekazu \\
\hline Citation & $\begin{array}{l}\text { Journal of Chemical Physics, 140(11), } 114302 \\
\text { https://doi.org/10.1063/1.4867909 }\end{array}$ \\
\hline Issue Date & $2014-03-21$ \\
\hline Doc URL & http:/hdl .handle.net/2115/56370 \\
\hline Rights & $\begin{array}{l}\text { Copyright } 2014 \text { A merican Institute of Phy sics. This article may be downloaded for personal use only. A ny other use } \\
\text { requires prior permission of the author and the A merican Institute of Physics. The following article appeared in J. } \\
\text { Chem. Phys. 140, } 114302 \text { (2014) and may be found at http://dx.doi.org/10.1063/1.4867909. }\end{array}$ \\
\hline Type & article \\
\hline File Information & JCP140-11_114302.pdf \\
\hline
\end{tabular}

Instructions for use 


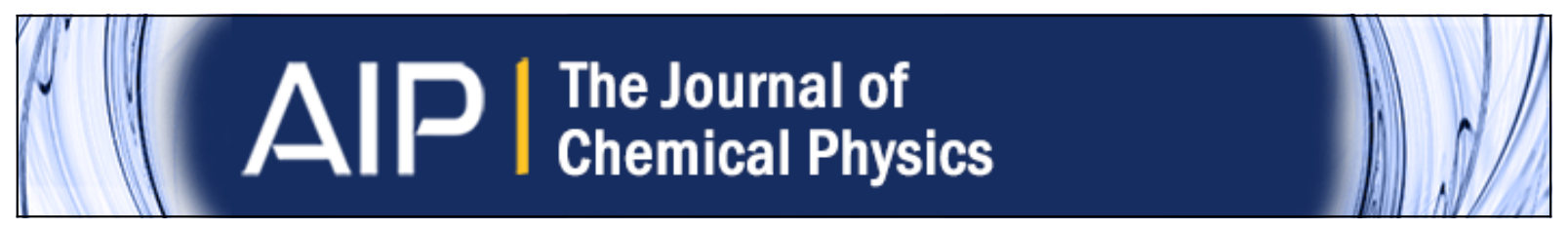

Molecular dynamics simulations of the nucleation of water: Determining the sticking probability and formation energy of a cluster

Kyoko K. Tanaka, Akio Kawano, and Hidekazu Tanaka

Citation: The Journal of Chemical Physics 140, 114302 (2014); doi: 10.1063/1.4867909

View online: http://dx.doi.org/10.1063/1.4867909

View Table of Contents: http://scitation.aip.org/content/aip/journal/jcp/140/11 ?ver=pdfcov

Published by the AIP Publishing

\section{Articles you may be interested in}

Free energy of cluster formation and a new scaling relation for the nucleation rate

J. Chem. Phys. 140, 194310 (2014); 10.1063/1.4875803

Properties of liquid clusters in large-scale molecular dynamics nucleation simulations

J. Chem. Phys. 140, 074303 (2014); 10.1063/1.4865256

Molecular dynamics simulations of nucleation from vapor to solid composed of Lennard-Jones molecules

J. Chem. Phys. 134, 204313 (2011); 10.1063/1.3593459

Cluster sizes in direct and indirect molecular dynamics simulations of nucleation

J. Chem. Phys. 131, 244511 (2009); 10.1063/1.3279127

Energy dependent decay rates of Lennard-Jones clusters for use in nucleation theory

J. Chem. Phys. 126, 074312 (2007); 10.1063/1.2698424

\section{AlP Re-register for Table of Content Alerts}




\title{
Molecular dynamics simulations of the nucleation of water: Determining the sticking probability and formation energy of a cluster
}

\author{
Kyoko K. Tanaka, ${ }^{1, a)}$ Akio Kawano, ${ }^{2}$ and Hidekazu Tanaka ${ }^{1}$ \\ ${ }^{1}$ Institute of Low Temperature Science, Hokkaido University, Sapporo 060-0819, Japan \\ ${ }^{2}$ Japan Agency for Marine-Earth Science and Technology, Kanagawa, Japan
}

(Received 13 September 2013; accepted 25 February 2014; published online 17 March 2014)

\begin{abstract}
We performed molecular dynamics simulations of the nucleation of water vapor in order to test nucleation theories. Simulations were performed for a wide range of supersaturation ratios $(S=3-25)$ and water temperatures $\left(T_{\mathrm{w}}=300-390 \mathrm{~K}\right)$. We obtained the nucleation rates and the formation free energies of a subcritical cluster from the cluster size distribution. The classical nucleation theory and the modified classical nucleation theory (MCNT) overestimate the nucleation rates in all cases. The semi-phenomenological model, which corrects the MCNT prediction using the second virial coefficient of a vapor, reproduces the formation free energy of a cluster with the size $\lesssim 20$ to within $10 \%$ and the nucleation rate and cluster size distributions to within one order of magnitude. The sticking probability of the vapor molecules to the clusters was also determined from the growth rates of the clusters. The sticking probability rapidly increases with the supersaturation ratio $S$, which is similar to the Lennard-Jones system. (C) 2014 AIP Publishing LLC. [http://dx.doi.org/10.1063/1.4867909]
\end{abstract}

\section{INTRODUCTION}

The classical nucleation theory (CNT) is the most widely used model for describing homogeneous nucleation and provides the nucleation rate as a function of the supersaturation ratio and the surface tension of a condensed phase. ${ }^{1-4}$ However, many studies have reported that the CNT fails to describe experimentally obtained results. ${ }^{5-27}$ In the case of water, the deviations between the nucleation rates predicted by the classical nucleation theory and the experimental values are in the order of $10^{2}-10^{3} .6,13,18,20-27$ The nucleation rates obtained by molecular dynamics (MD) and Monte Carlo (MC) simulations also significantly differ from the predictions of the CNT. ${ }^{28-49}$ The nucleation rate is governed by the formation free energy of a critical cluster, which is the smallest thermodynamically stable cluster. In the classical nucleation theory, the formation free energy is simply evaluated using the surface energy of the bulk material. Since the critical clusters are considered to be nano-sized, the error in the classical theory is thought to come from the difference in the properties of such a small cluster and a bulk material.

In previous studies, there have been significant advances in theoretical models of homogeneous nucleation., ${ }^{9}, 10,12,14,40,50-55$ One of the most successful and useful models is the semi-phenomenological (SP) model, $,{ }^{9}, 12,14$ which corrects the evaluation of the formation energy of a cluster in the CNT by using the second virial coefficient of a vapor. The predictions of the SP model agree well with experimental data for various substances including water., ${ }^{9}, 14$ By performing MD simulations of nucleation for Lennard-Jones systems, Tanaka et al. ${ }^{46,48}$ tested the SP model and found that it also agrees well with their MD simulations. However, only large supersaturation ratios were possible

\footnotetext{
${ }^{a)}$ Electronic mail: kktanaka@lowtem.hokudai.ac.jp
}

in their MD simulations. Recent large scale simulations by Diemand et al. ${ }^{49}$ found some deviations in the nucleation rate from the SP model in the case of a small supersaturation ratio.

Besides Lennard-Jones systems, many other MD simulations examining the nucleation process of water molecules have been carried out. Yasuoka and Matsumoto ${ }^{31}$ investigated homogeneous nucleation for the first time using MD simulations of water molecules. The nucleation rate they obtained at $350 \mathrm{~K}$ was two orders of magnitude less than that predicted by the classical theory. Matsubara et al. ${ }^{47}$ carried out MD simulations at various temperatures and supersaturation ratios, using a simple point charge/extended (SPC/E) water model. ${ }^{56}$ They measured the nucleation rate, the critical nucleus size, and the formation free energy of a cluster and compared them with the various theoretical models. They showed that all theoretical models (CNT, SP model, and the scaled model) predict the nucleation rates obtained by the simulations to within one or two orders of magnitude. On the other hand, the formation free energy of a cluster derived by the simulations was considerably larger than that obtained with the theoretical models. They showed that the deviations in the formation free energy can be explained by the larger growth rate of clusters than that assumed by the theories. Recently, Zipoli et al. ${ }^{57}$ developed a new coarse-grained model for water to study nucleation from the vapor and obtained smaller nucleation rates than the previous studies.

In addition to the formation free energy of a cluster, the sticking probability $\alpha$ of vapor molecules to clusters is another important factor for determining the nucleation rate. The sticking probability is usually assumed to be unity, despite the fact that the nucleation rate is proportional to $\alpha$ in the nucleation theory. In the previous studies, ${ }^{48,49}$ the sticking probability in a Lennard-Jones type system was examined through MD simulations, by observing the growth rate of stable 
clusters larger than the critical size. They showed that the sticking probability decreases with decreasing supersaturation ratio; however, the validity of their findings has not been confirmed. Therefore, it is worthwhile investigating the sticking probability for various materials.

In the present study, we performed MD simulations of water molecules for a wide range of initial supersaturation ratios and temperatures. We observed the nucleation rates and derived the formation free energy by using the size distribution of the clusters. By comparing these numerical results with theories, we can test the theoretical models. We also obtained the sticking probability from the MD simulations based on the previous method. ${ }^{48,49}$ In Sec. II, we describe the numerical procedure for our MD simulations, and in Sec. III we present our numerical results and compare the numerical results with the theories. We also determine the sticking probability and the dependence of the supersaturation ratio. In Sec. IV, we summarize the results of the present study.

\section{NUMERICAL PROCEDURE AND ANALYSIS}

We performed simulations of the nucleation process in systems of 4000 of water molecules, using the SPC/E rigid water model. ${ }^{56}$ We assumed $N V T$ (constant volume and temperature) ensembles and used a three-dimensional periodic boundary condition. Initially, the molecules are located randomly without being overlapped with others and the system is relaxed to be in the equilibrium state at $1000 \mathrm{~K}$. Using this initial condition, we started the simulations. In our simulations, the number of water molecules was not so large. In order to improve the statistical accuracy, we performed 20 runs with different initial positions and velocities of molecules for each parameters of temperature $T$ and initial supersaturation ratio $S_{0}$. The time step was set as $2.0 \mathrm{fs}$. The total number of time steps was $(4-10) \times 10^{6}$ in each run. The simulation box contained 4000 water molecules and 4000 (or 8000) argon carrier gas molecules to control the temperature. To compute the long-range electrostatic interactions, we adopted the reaction field-zero method ${ }^{58}$ in which a dielectric constant of infinity and a group-based cutoff are employed. In this study we set the cutoff radius to $3.5 \mathrm{~nm}$.

The computational region is a cube with periodic boundaries. By varying the box size $L$, we set the initial number density of molecules or the initial supersaturation ratio $S_{0}$. The supersaturation ratio is defined as $S=P_{1} / P_{\text {sat }}$, where $P_{1}$ and $P_{\text {sat }}$ are the partial pressure of the monomers and the pressure in a saturated vapor. The resulting supersaturation is approximately given by $S=P_{\text {total }} / P_{\text {sat }}$ with the total pressure $P_{\text {total }}$ because of small amount of clusters larger than dimers. Simulations were performed for various temperatures and supersaturation ratios, i.e., $T=250-375 \mathrm{~K}$ and $S=3-25$. The parameter sets for each run are shown in Table I. Twenty-eight parameter sets were chosen, so that the total number of simulations is 560. For a sufficiently high supersaturation ratio, the simulation gives a very large nucleation rate and the number of the vapor molecules decreases so quickly. In such a case, a steady nucleation with a constant supersaturation ratio is not realized. In our simulations, in order to observe steady nucleation, we chose lower supersaturation ratios compared with
TABLE I. Parameters in the MD simulations, temperature of carrier gas $T$, cell size $L$, initial number density of monomers $n(1)_{t=0}$, number of carrier gas $N_{\text {cg }}$, and simulation time $t_{\text {end }}$.

\begin{tabular}{|c|c|c|c|c|c|}
\hline Run \# & $T(\mathrm{~K})$ & $L(\mathrm{~nm})$ & $\begin{array}{c}n(1)_{t=0} \\
{\left[10^{-2} \mathrm{~nm}^{-3}\right]}\end{array}$ & $N_{\text {cg }}$ & $t_{\text {end }}(\mathrm{ns})$ \\
\hline $6 e$ & 375 & 44.10 & 4.66 & 4000 & 20 \\
\hline $6 \mathrm{~d}$ & 375 & 42.30 & 5.28 & 4000 & 15 \\
\hline $6 c$ & 375 & 40.50 & 6.02 & 4000 & 10 \\
\hline $6 b$ & 375 & 36.82 & 8.01 & 4000 & 8 \\
\hline $5 e$ & 350 & 52.50 & 2.76 & 4000 & 30 \\
\hline $5 \mathrm{e} 2$ & 350 & 52.50 & 2.76 & 8000 & 30 \\
\hline $5 d$ & 350 & 49.50 & 3.30 & 4000 & 15 \\
\hline $5 c$ & 350 & 45.00 & 4.39 & 4000 & 10 \\
\hline $5 b$ & 350 & 40.91 & 5.84 & 4000 & 8 \\
\hline $4 \mathrm{e}$ & 325 & 59.90 & 1.86 & 4000 & 20 \\
\hline $4 \mathrm{e} 2$ & 325 & 59.90 & 1.86 & 8000 & 20 \\
\hline $4 d$ & 325 & 54.45 & 2.48 & 4000 & 15 \\
\hline $4 c$ & 325 & 49.50 & 3.30 & 4000 & 10 \\
\hline $4 b$ & 325 & 45.00 & 4.39 & 4000 & 8 \\
\hline $3 e$ & 300 & 65.34 & 1.43 & 4000 & 20 \\
\hline $3 \mathrm{e} 2$ & 300 & 65.34 & 1.43 & 8000 & 20 \\
\hline $3 d$ & 300 & 59.40 & 1.91 & 4000 & 15 \\
\hline $3 c$ & 300 & 54.00 & 2.54 & 4000 & 10 \\
\hline $3 b$ & 300 & 49.09 & 3.38 & 4000 & 8 \\
\hline $2 \mathrm{e}$ & 275 & 70.79 & 1.13 & 4000 & 20 \\
\hline $2 \mathrm{e} 2$ & 275 & 70.79 & 1.13 & 8000 & 20 \\
\hline $2 d$ & 275 & 64.35 & 1.50 & 4000 & 15 \\
\hline $2 c$ & 275 & 58.50 & 2.00 & 4000 & 10 \\
\hline $2 b$ & 275 & 53.18 & 2.66 & 4000 & 8 \\
\hline $1 \mathrm{e}$ & 250 & 76.23 & 0.903 & 4000 & 20 \\
\hline $1 d$ & 250 & 69.30 & 1.20 & 4000 & 15 \\
\hline $1 \mathrm{c}$ & 250 & 63.00 & 1.60 & 4000 & 10 \\
\hline $1 b$ & 250 & 57.27 & 2.13 & 4000 & 8 \\
\hline
\end{tabular}

the previous study.$^{47}$ For the interaction between carrier gas and water, we used the potential of the Lennard-Jones type:

$$
V(r)=4 \epsilon\left[(\sigma / r)^{12}-(\sigma / r)^{6}\right],
$$

where $r$ is the distance between argon and oxygen, and the parameters $\sigma$ and $\epsilon$ are given by $\sigma=\sqrt{\sigma_{\mathrm{Ar}} \sigma_{\mathrm{O}}}$ and $\epsilon=\sqrt{\varepsilon_{\mathrm{Ar}} \varepsilon_{\mathrm{O}}}$ with $\sigma_{\mathrm{Ar}}=3.40 \AA, \sigma_{\mathrm{O}}=3.17 \AA, \varepsilon_{\mathrm{Ar}}$ $=0.979 \mathrm{~kJ} \mathrm{~mol}^{-1}$, and $\varepsilon_{\mathrm{O}}=0.652 \mathrm{~kJ} \mathrm{~mol}^{-1} .{ }^{59}$ The carrier gas temperature $T$ was controlled directly with a Nosé-Hoover thermostat, while the temperature of the water was controlled indirectly through interaction with the carrier gas molecules. The temperature of the water molecules $T_{\mathrm{w}}$ deviates from the temperature of the carrier gas because of the latent heat effect that results from condensation formation. We defined clusters using the bonding criterion that the interaction energy of a molecular pair is less than $-10 \mathrm{~kJ} / \mathrm{mol}^{47}$

We obtained the nucleation rate and compared the number density of clusters based on the same method as in the previous studies. ${ }^{48,49}$ We also derived the formation free energies from the cluster size distribution. ${ }^{60}$ To derive the formation free energy of a cluster $\Delta G_{i}$, where $i$ is the number of molecules in the cluster, we use the relation between the equilibrium size distribution $n_{\mathrm{e}}(i)$ and the formation free energy of 
a cluster $\Delta G_{i}:{ }^{48,49}$

$$
\frac{\Delta G_{i}}{k T}=\ln \left(\frac{n(1)}{n_{\mathrm{e}}(i)}\right),
$$

where $n(1)$ is the number density of the monomers.

The steady nucleation rate $J$ is the net number of the transition from $i$-mer to $i+1$-mer and given by

$$
J=R^{+}(i) n(i)-R^{-}(i+1) n(i+1),
$$

where $R^{+}(i)$ is the transition rate from a cluster of $i$ molecules, $i$-mer, to $(i+1)$-mer per unit time, i.e., the accretion rate, and $R^{-}(i)$ is the transition rate from $i$-mer to $(i-1)$-mer per unit time, i.e., the evaporation rate of $i$-mer. $R^{+}(i)$ is given by

$$
R^{+}(i)=\alpha n(1) v_{\mathrm{th}}\left(4 \pi r_{0}^{2} i^{2 / 3}\right),
$$

where $v_{\text {th }}$ is the thermal velocity, $\sqrt{k T / 2 \pi m}$, and $r_{0}$ is the radius of a monomer, $\left(3 m / 4 \pi \rho_{\mathrm{m}}\right)^{1 / 3}$, where $m$ is the mass of a molecule and $\rho_{\mathrm{m}}$ is the bulk density. The evaporation rate is obtained from the principle of detailed balance in thermal equilibrium:

$$
R^{-}(i+1) n_{\mathrm{e}}(i+1)=R^{+}(i) n_{\mathrm{e}}(i)
$$

From Eqs. (4) and (5), we have

$$
J=R^{+}(i) n(i)-R^{+}(i) n_{\mathrm{e}}(i) \frac{n(i+1)}{n_{\mathrm{e}}(i+1)},
$$

which leads to

$$
\frac{n_{\mathrm{e}}(i)}{n(i)}=\frac{n_{\mathrm{e}}(i-1)}{n(i-1)}\left(1-\frac{J}{R^{+}(i-1) n(i-1)}\right)^{-1} .
$$

Equation (7) shows $n_{\mathrm{e}}(i)$ agrees well with the number density of the clusters $n(i)$ obtained by the MD simulations for subcritical clusters $i \leq i^{*}$, because $J \ll R^{+}(i) n(i)$ for $i \leq i^{*}$. From Eq. (7), we obtain $n_{\mathrm{e}}(i)$ if $J, n(i)$ and $n_{\mathrm{e}}(i-1)$ are given. For example, $n_{\mathrm{e}}(2)$ is obtained by $J, n(2)$, and $n_{\mathrm{e}}(1)[=n(1)]$. Also for a larger $i$, we can obtain $n_{\mathrm{e}}(i)$ recursively. Matsubara et $a{ }^{47}{ }^{47}$ calculated the equilibrium number density, using the different formula derived by Yasuoka and Matsumoto ${ }^{30}$ from Fokker-Plank equation. Our formula leads to the same as Matsubara et $a l^{47}$ for $i^{*} \gg 1$ (see the Appendix). by

In the theoretical models, the formulae for $\Delta G_{i}$ are given

$$
\begin{aligned}
\frac{\Delta G_{\mathrm{CNT}, \mathrm{i}}}{k T} & =-i \ln S+\eta i^{2 / 3}, \\
\frac{\Delta G_{\mathrm{MCNT}, \mathrm{i}}}{k T} & =-(i-1) \ln S+\eta\left(i^{2 / 3}-1\right), \\
\frac{\Delta G_{\mathrm{SP}, \mathrm{i}}}{k T} & =-(i-1) \ln S+\eta\left(i^{2 / 3}-1\right)+\xi\left(i^{1 / 3}-1\right),
\end{aligned}
$$

where $\Delta G_{\mathrm{CNT}, \mathrm{i}}, \Delta G_{\mathrm{MCNT}, \mathrm{i}}$, and $\Delta G_{\mathrm{SP}, \mathrm{i}}$ are the formation free energies of a cluster in the CNT, the modified CNT (MCNT), and the SP model, respectively, and $\eta$ and $\xi$ are parameters determined by the bulk surface energy and second virial coefficient. ${ }^{48,49}$ In the classical theory, there is a theoretical inconsistency in the $S$ dependence. Consequently the MCNT has often been used. In Sec. III, we compare the CNT, MCNT, and SP models with the our numerical results. Although there are some differences between the thermodynamic quantities obtained by using SPC/E force and those by experiments of real water, we should use those of SPC/E force for the consistency to the model. For thermodynamic quantities such as the surface tension and the saturated vapor pressure of the SPC/E water, we use the same data as Matsubara et al. ${ }^{47}$

\section{RESULTS}

\section{A. A typical case}

Let $N\left(>i_{\text {th }}\right)$ denote the number of clusters larger than a threshold size $i_{\text {th }}$. Based on Yasuoka and Matsumoto method, ${ }^{30}$ we can obtain the nucleation rate from $N\left(>i_{\text {th }}\right)$. Figure 1 shows time evolution of the supersaturation ratio and the number of clusters, $N\left(>i_{\text {th }}\right)$, in a typical case where $T$ $=350 \mathrm{~K}$ and $S_{0}=6.67$ (run $5 \mathrm{~d}$ in Table I). Note that $N\left(>i_{\text {th }}\right)$ is averaged over 20 runs with different initial positions and velocities of molecules. In Figure 1, we plot $N\left(>i_{\text {th }}\right)$ for $i_{\text {th }}=20,30$, and 40 .

The threshold $i_{\text {th }}$ should be larger than the critical size $i^{*}$ for the evaluation of the nucleation rate. In this case, the critical size $i^{*}$ is estimated to be 14 (or 7) with the SP (or CNT) model. In this figure, the number of stable nuclei $N\left(>i_{\text {th }}\right)$ increases almost linearly in the period of $t=(6-$ 12) ns. From the slope, the nucleation rate is measured with $J\left(=\dot{N}\left[>i_{\text {th }}\right] / L^{3}\right)$. In the evaluation of $\mathrm{J}$, we chose the slope between the times at $N\left(>i_{\text {th }}\right)=2$ and 4, e.g., $t=8-10 \mathrm{~ns}$ for $i_{\text {th }}=20$. Then, the nucleation rate is obtained as $8.92 \times 10^{24}$, $7.37 \times 10^{24}$, and $6.19 \times 10^{24} \mathrm{~cm}^{-3} \mathrm{~s}^{-1}$, for $i_{\text {th }}=20,30$, and 40 , respectively. In this evaluation of the nucleation rate, uncertainties of 20\%-30\% exist, depending on the choice of $i_{\text {th }}$ and the time interval.

During this period of $t=(8-10) \mathrm{ns}$, the average value of the supersaturation $S$ and the temperature of the water

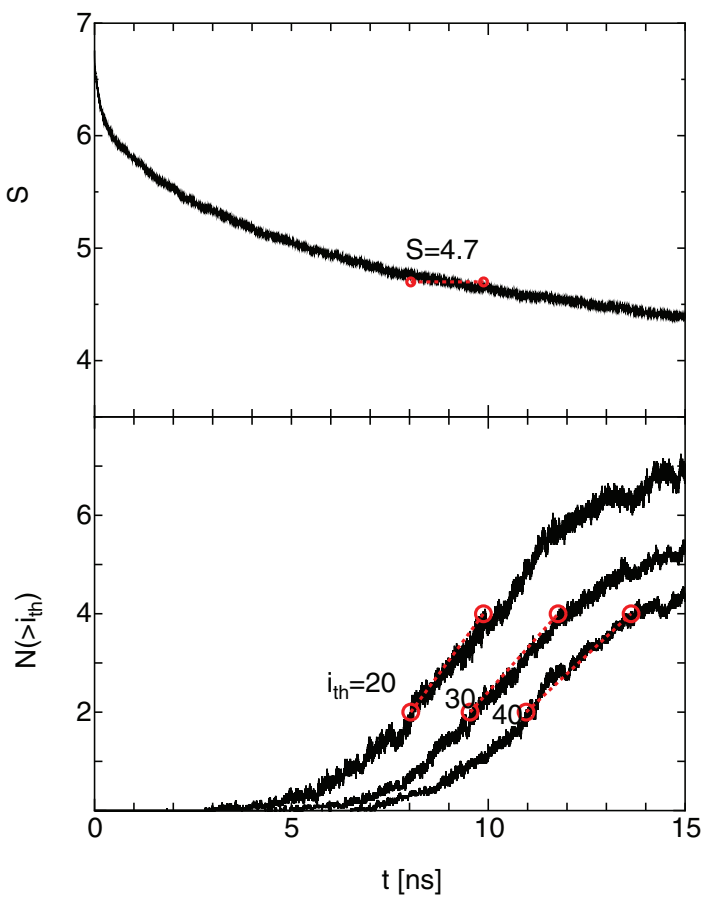

FIG. 1. Supersaturation ratio and number of clusters above various threshold sizes as a function of time for run $5 \mathrm{~d}$, where $N\left(>i_{\text {th }}\right)$ is averaged from 20 runs with the same initial conditions. 
molecules $T_{\mathrm{w}}$ were 4.7 and $361 \mathrm{~K}$, respectively. Using these values, we can calculate $J$ predicted by the theoretical models. In the MCNT, the CNT, and the SP models, the nucleation rates are obtained as $1.5 \times 10^{27}, 8.0 \times 10^{25}$, and 2.1 $\times 10^{24} \mathrm{~cm}^{-3} \mathrm{~s}^{-1}$, respectively. The prediction of the SP model is much closer to the values obtained in the MD simulations than the MCNT and the CNT.

\section{B. Nucleation rate}

Fig. 2 shows the time evolutions of $N\left(>i_{\mathrm{th}}\right)$ for $i_{\mathrm{th}}=20$, 30 , and 40 in the various runs $(6 e, 5 c, 3 c$, and $2 \mathrm{e})$. We evaluate the nucleation rates from the slopes between the times at $N\left(>i_{\mathrm{th}}\right)=2$ and $N\left(>i_{\mathrm{th}}\right)=4$ for all runs except run $6 \mathrm{e}$. For run $6 \mathrm{e}$, we use the slope between the times at $N\left(>i_{\mathrm{th}}\right)=1$ and 3 because of the small number of clusters. The nucleation rates obtained in all MD simulations are listed in Table II, where we chose $i_{\text {th }}=20$ for all runs because the size of critical cluster is estimated to be less than 20 for all runs (see Sec. III D). As stated in Sec. III A, there are some uncertainties in the estimation of the nucleation rates. So we include the errors in the nucleation rates in Table II.

Fig. 3 shows the nucleation rates obtained by the MD simulations as a function of the supersaturation ratio for various temperatures. The previous results are also shown: crosses and triangles are the results by Matsubara et $a l .{ }^{47}$ and Zipoli et al. ${ }^{57}$ respectively. The nucleation rates by SP model (solid

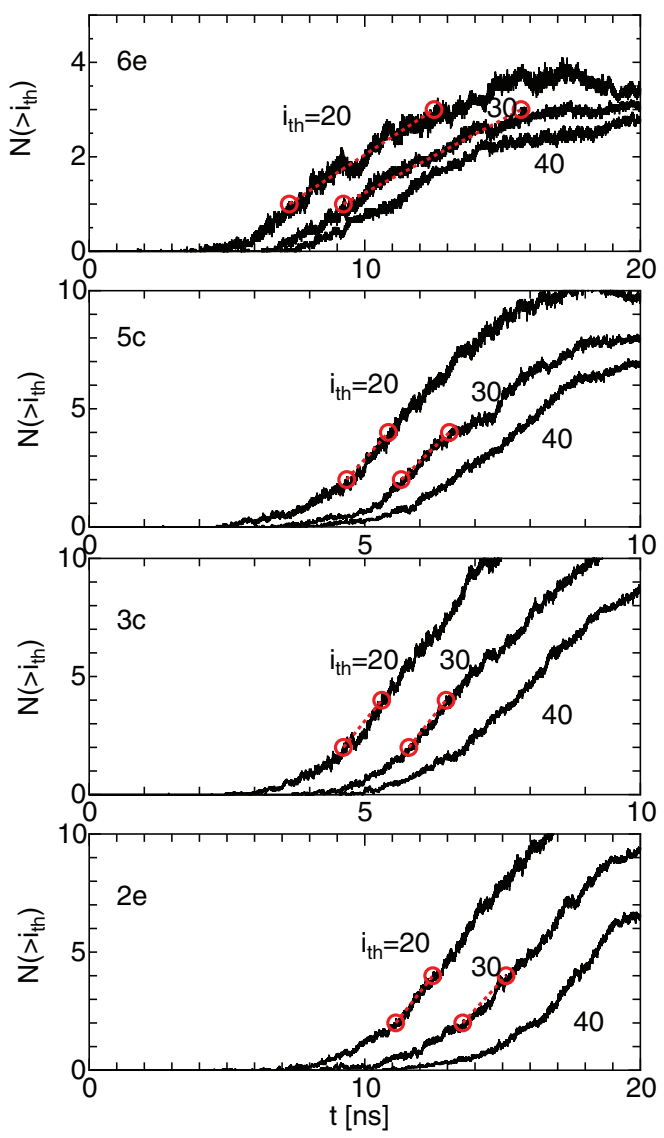

FIG. 2. Number of clusters above various threshold sizes as a function of time for various runs, where $N\left(>i_{\text {th }}\right)$ is averaged from 20 runs with the same initial conditions.

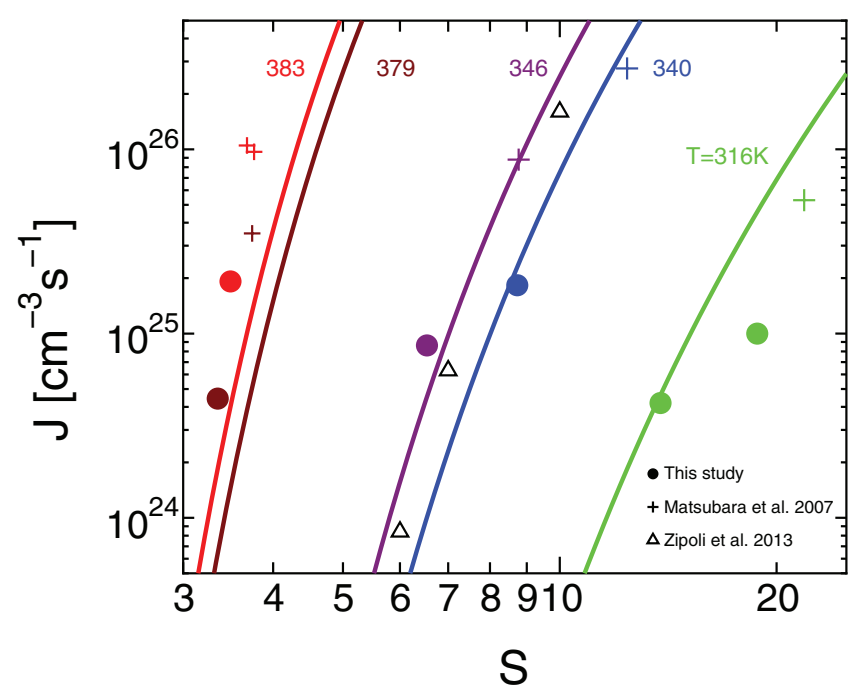

FIG. 3. The nucleation rates obtained by the MD simulations as a function of the supersaturation ratio for various temperatures. The previous results are also shown: crosses and triangles are the results by Matsubara et al. ${ }^{47}$ and Zipoli et al.,${ }^{57}$ respectively. In Zipoli et al. ${ }^{57}$ we use the nucleation rates at $350 \mathrm{~K}$. The nucleation rates by SP model (solid curve) are also plotted.

curves) are also plotted. The obtained nucleation rates are about one-order-of-magnitude smaller than Matsubara et al. ${ }^{47}$ because of the smaller supersaturation ratios. We find good agreements between the MD simulations and the SP model within one-order-of-magnitude in the cases of low temperatures for both our runs and the previous results.

Figure 4 shows the ratios of the nucleation rates in the MD simulations (at $T_{\mathrm{w}}$ ) to the rates obtained with the theoretical models. The results are plotted with circles for the SP model, triangles for the MCNT, and crosses for the CNT. The nucleation rates predicted by the SP model are within one order of magnitude of the MD simulations, whereas the nucleation rates predicted by the CNT and MCNT are much larger than the MD simulations. We also plotted the ratios $J_{\mathrm{MD}} / J_{\mathrm{SP}}$ by using results by Matsubara et $a{ }^{47}$ (i.e., data in Table I in Ref. 47). Our results agree well with theirs. The ratio of $J_{\mathrm{MD}} / J_{\mathrm{SP}}$ gradually increases with temperature in both results. The nucleation rates obtained with the theoretical models are also listed in Table II.

In Figure 4, we also show the results of the LennardJones system $^{48}$ for reference. The SP model also predicts the nucleation rates to within one order of magnitude for the Lennard-Jones system, although the supersaturation ratios are limited to be small. In the case of the Lennard-Jones system, the CNT considerably underestimates the nucleation rates (i.e., the $J_{\mathrm{MD}} / J_{\mathrm{CNT}}$ ratio is much larger than $10^{9}$ ) at $T$ $<0.4\left(\varepsilon / k_{\mathrm{B}}\right)$, where $\varepsilon$ and $k_{\mathrm{B}}$ are the depth of the LennardJones potential and the Boltzmann constant, respectively. On the other hand, the CNT predictions agree with the nucleation rates obtained by the MD simulations to within two orders of magnitude in the case of water.

In Fig. 5, we show the nucleation rates as a function of $\ln S /\left(T_{c} / T-1\right)^{1.5}$ in order to compare our results with the scaling relation proposed by Hale. ${ }^{61}$ The data by the MD simulations and the theoretical predictions are the same as those in Fig. 3. For the MD simulations using SPC/E water model, we 
TABLE II. Summary of the results of the MD simulations. $T$ [K]: temperature of carrier gas, $T_{\mathrm{w}}[\mathrm{K}]$ : temperature of water molecules, $S$ : averaged supersaturation ratio in the nucleation stage, $J_{\mathrm{MD}}\left[10^{24} \mathrm{~cm}^{-3} \mathrm{~s}^{-1}\right]$ : nucleation rates obtained by the MD simulations, $J_{\mathrm{SP}}\left[10^{24} \mathrm{~cm}^{-3} \mathrm{~s}^{-1}\right]$ : nucleation rates by the SP model, $J_{\mathrm{MCNT}}\left[10^{24} \mathrm{~cm}^{-3} \mathrm{~s}^{-1}\right]$ : nucleation rates by the MCNT, $J_{\mathrm{CNT}}\left[10^{24} \mathrm{~cm}^{-3} \mathrm{~s}^{-1}\right]$ : nucleation rates by the CNT, $i^{*}$ : critical cluster size evaluated by the MD simulations, $i_{\mathrm{SP}}^{*}$ : critical cluster size evaluated by the SP model, $i_{\mathrm{CNT}}^{*}$ : critical cluster size evaluated by the CNT (or MCNT) model, and $\alpha$ : sticking probability.

\begin{tabular}{|c|c|c|c|c|c|c|c|c|c|c|c|}
\hline Run \# & $T$ & $T_{\mathrm{W}}$ & $S$ & $J_{\mathrm{MD}}$ & $J_{\mathrm{SP}}$ & $J_{\mathrm{MCNT}}$ & $J_{\mathrm{CNT}}$ & $i^{*}$ & $i_{\mathrm{SP}}^{*}$ & $i_{\mathrm{CNT}}^{*}$ & $\alpha$ \\
\hline $6 e$ & 375 & 379 & 3.35 & $4.44 \pm 0.82$ & 0.563 & 985 & 58.4 & 14 & 20 & 10 & 0.328 \\
\hline $6 d$ & 375 & 380 & 3.37 & $9.74 \pm 1.6$ & 1.13 & 1461 & 92.1 & 14 & 19 & 10 & 0.425 \\
\hline $6 c$ & 375 & 383 & 3.49 & $19.2 \pm 2.3$ & 3.71 & 2811 & 193.1 & 13 & 17 & 9 & 0.479 \\
\hline $6 \mathrm{~b}$ & 375 & 390 & 3.41 & $83.7 \pm 19.0$ & 13.7 & 6116 & 490.8 & 12 & 16 & 8 & 0.671 \\
\hline $5 e$ & 350 & 357 & 4.61 & $3.71 \pm 0.65$ & 0.67 & 822.1 & 38.5 & 13 & 15 & 7 & 0.407 \\
\hline $5 e-2$ & 350 & 355 & 4.98 & $7.03 \pm 3.0$ & 1.29 & 1189 & 53.5 & 11 & 14 & 6 & 0.464 \\
\hline $5 d$ & 350 & 361 & 4.70 & $8.92 \pm 1.5$ & 2.05 & 1530 & 79.5 & 12 & 14 & 7 & 0.472 \\
\hline $5 c$ & 350 & 366 & 4.78 & $29.1 \pm 3.9$ & 9.77 & 3684 & 224.7 & 14 & 13 & 6 & 0.609 \\
\hline $5 b$ & 350 & 374 & 4.50 & $68.7 \pm 9.6$ & 27.2 & 7015 & 497.0 & 13 & 12 & 6 & 0.798 \\
\hline $4 \mathrm{e}$ & 325 & 340 & 6.72 & $3.73 \pm 0.8$ & 1.46 & 911 & 38.0 & 11 & 11 & 5 & 0.476 \\
\hline $4 e-2$ & 325 & 338 & 7.28 & $5.94 \pm 0.3$ & 2.32 & 1099 & 46.7 & 12 & 10 & 5 & 0.649 \\
\hline $4 d$ & 325 & 346 & 6.54 & $8.63 \pm 0.5$ & 4.79 & 1837 & 88.6 & 12 & 11 & 5 & 0.613 \\
\hline $4 c$ & 325 & 352 & 6.48 & $19.1 \pm 4.3$ & 16.8 & 3805 & 213 & 11 & 10 & 4 & 0.719 \\
\hline $4 \mathrm{~b}$ & 325 & 359 & 6.08 & $49.1 \pm 9.8$ & 39.6 & 6630 & 422 & 11 & 10 & 4 & 0.904 \\
\hline $3 e$ & 300 & 328 & 10.0 & $2.99 \pm 0.4$ & 3.7 & 1076 & 43.9 & 10 & 9 & 4 & 0.622 \\
\hline $3 e-2$ & 300 & 323 & 11.8 & $4.92 \pm 0.5$ & 7.08 & 1347 & 57.3 & 10 & 8 & 3 & 0.762 \\
\hline $3 d$ & 300 & 334 & 9.09 & $7.42 \pm 0.3$ & 8.14 & 1845 & 84.7 & 11 & 9 & 4 & 0.794 \\
\hline $3 c$ & 300 & 340 & 8.73 & $18.3 \pm 0.57$ & 21.5 & 3369 & 175.8 & 9 & 8 & 3 & 0.776 \\
\hline $3 b$ & 300 & 347 & 7.99 & $46.6 \pm 21$ & 47.8 & 5813 & 343 & 10 & 8 & 3 & 0.938 \\
\hline $2 e$ & 275 & 316 & 13.8 & $4.20 \pm 0.60$ & 4.69 & 921.8 & 36.4 & 9 & 7 & 3 & 0.748 \\
\hline $2 \mathrm{e}-2$ & 275 & 310 & 18.6 & $4.50 \pm 0.60$ & 12.2 & 1254 & 53.7 & 10 & 6 & 2 & 1.02 \\
\hline $2 d$ & 275 & 322 & 13.4 & $5.59 \pm 1.1$ & 13.5 & 1766 & 79.6 & 11 & 7 & 3 & 0.803 \\
\hline $2 \mathrm{c}$ & 275 & 327 & 12.7 & $16.1 \pm 4.7$ & 33.2 & 3144 & 160 & 10 & 7 & 3 & 0.911 \\
\hline $2 b$ & 275 & 335 & 11.2 & $20.0 \pm 4.3$ & 62.4 & 5113 & 287 & 11 & 7 & 3 & 1.02 \\
\hline $1 \mathrm{e}$ & 250 & 303 & 23.0 & $4.39 \pm 2.0$ & 9.47 & 908 & 37.2 & 9 & 6 & 2 & 0.885 \\
\hline $1 d$ & 250 & 310 & 21.0 & $5.22 \pm 0.95$ & 2.19 & 1623 & 74.1 & 10 & 6 & 2 & 0.927 \\
\hline $1 \mathrm{c}$ & 250 & 316 & 18.8 & $10.0 \pm 1.4$ & 42.1 & 2644 & 132 & 10 & 6 & 2 & 1.01 \\
\hline $1 \mathrm{~b}$ & 250 & 323 & 16.3 & $24.0 \pm 3.1$ & 82.6 & 4472 & 246 & 10 & 6 & 2 & 1.12 \\
\hline
\end{tabular}

set $T_{c}=630 \mathrm{~K}$ for the consistency to the model. ${ }^{47} \mathrm{We}$ also put the experimental data for water nucleation. $6,20,22,23,26,27$ For the experimental results and Zipoli et al. ${ }^{57} T_{c}$ is set to be $647 \mathrm{~K} .{ }^{20}$ From Fig. 5, $J$ seems to be scaled by $\ln S /\left(T_{c} / T\right.$ $-1)^{1.5}$, but is not proportional to it.

\section{Cluster size distributions}

Figure 6 shows the size distributions $n(i)$ obtained from 8 runs at various temperatures $T_{\mathrm{w}}$ and initial supersaturation ratios. For each temperature, we show high- $S$ cases (right column) and low- $S$ cases (left column). The MCNT significantly overestimates the distributions (by factors $10^{3}$ ). In all cases, the SP model predicts the size distributions more accurately than both the MCNT and the CNT for clusters smaller than the critical size. At $i=2$ the SP model agrees perfectly with the MD simulations in all cases. However, the SP model disagrees with the results of the MD simulations for large clusters. There is also a relatively large deviation at $i=4$. This is due to the stable four-ring structure.$^{54,62}$ These results for the size distributions are consistent with the nucleation rate, as shown in Sec. III B.

\section{Formation free energy}

We derive the formation free energy of a cluster $\Delta G_{i}(S)$ using the equilibrium size distribution $n_{\mathrm{e}}(i)$ given by Eq. (7). In the derivation, we use the nucleation rate $J$ and $n(i)$ obtained by the MD simulations and $R^{+}(i)$, where we use the value of $\alpha$ measured by the simulations (see Sec. III E and Table II). Using the $S$-dependence of $\Delta G_{i}$ in Eq. (8) except the CNT, we also obtain $\Delta G_{i}$ at $S=1$ as

$$
\Delta G_{i}(S=1)=\Delta G_{i}(S)+(i-1) k T \ln S
$$

where $\Delta G_{i}$ is obtained from Eq. (2). $\Delta G_{i}(S=1)$ corresponds to the contribution of the surface effect to the formation free energy of a cluster. If we know $\Delta G_{i}(S=1)$, we can predict $\Delta G_{i}$ and the nucleation rate for any value of $S$.

Fig. 7 shows the size distribution of clusters obtained by MD simulations and the equilibrium one in run $5 \mathrm{~d}$. The formation free energies $\Delta G_{i}$ are shown for $S=4.7$ and $S=1$ in Fig. 7. From $\Delta G_{i}$ for $S=4.7$, we obtain the maximum value of the formation free energy and the size of the critical cluster in run 5d, i.e., $\Delta G_{i}^{*}=7.0 k T$ and $i_{*}=12$. Table II shows the sizes of critical cluster obtained by the simulations for all runs. 

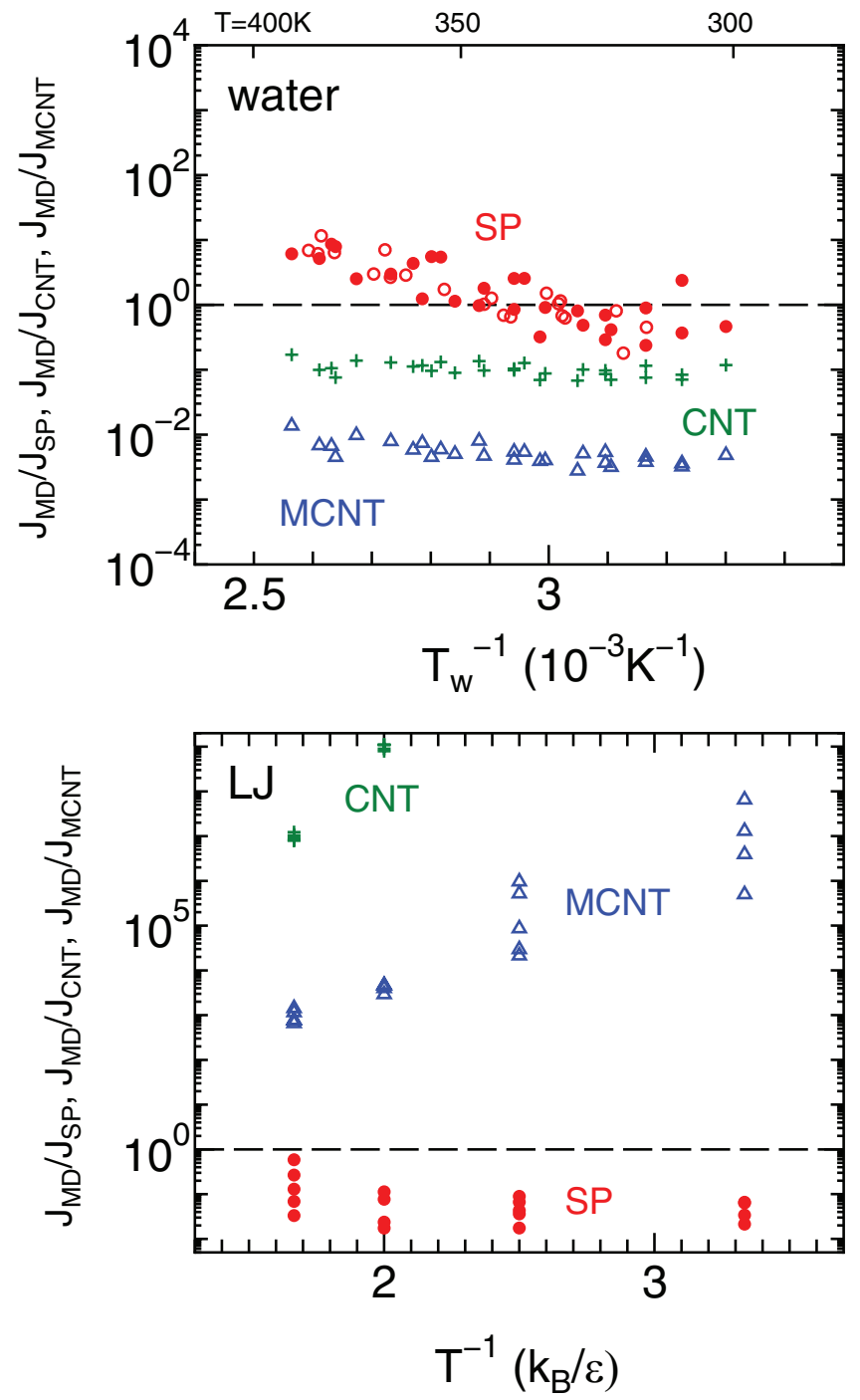

FIG. 4. The top panel shows the results for water obtained in this study. The ratios of nucleation rates of our MD simulations and the theoretical models plotted with closed circles for the SP model $\left(J_{\mathrm{MD}} / J_{\mathrm{SP}}\right)$, triangles for the $\operatorname{MCNT}\left(J_{\mathrm{MD}} / J_{\mathrm{MCNT}}\right)$, and crosses for the $\mathrm{CNT}\left(J_{\mathrm{MD}} / J_{\mathrm{CNT}}\right)$. The temperature is $T_{\mathrm{w}}$. By using $J_{\mathrm{MD}}$ obtained by Matsubara et al., ${ }^{47}$ we also plot $J_{\mathrm{MD}} / J_{\mathrm{SP}}$ with open circles. The results of the Lennard-Jones system ${ }^{48}$ are also shown in the bottom panel for reference.

In Fig. 8, we compare the maximum value of the formation free energy at the critical cluster between the theoretical models and the MD simulations for all runs. Evaluating $\Delta G_{i}^{*}$ in the theoretical model, we use the values of the temperature $T_{\mathrm{w}}$ and the supersaturation ratio obtained by the simulations shown in Table II. The CNT and MCNT considerably underestimate the maximum free energies. The SP model predicts the maximum free energies to a higher accuracy than the CNT and MCNT: the SP model predicts the maximum free energies within $30 \%$. In Matsubara et al.,$^{47}$ the similar comparison was performed. There are some differences between our results and Ref. 47. We confirmed that there is an inconsistency in Matsubara $e t ~ a l .{ }^{47}$ where they used slightly smaller temperatures in their figures than the true values of $T_{\mathrm{w}}$ in Table $\mathrm{I}^{47}$ (private communications). ${ }^{63}$

Figure 9 shows the formation free energy $\Delta G_{i}(S=1)$ obtained by simulations at various temperatures. Predictions of

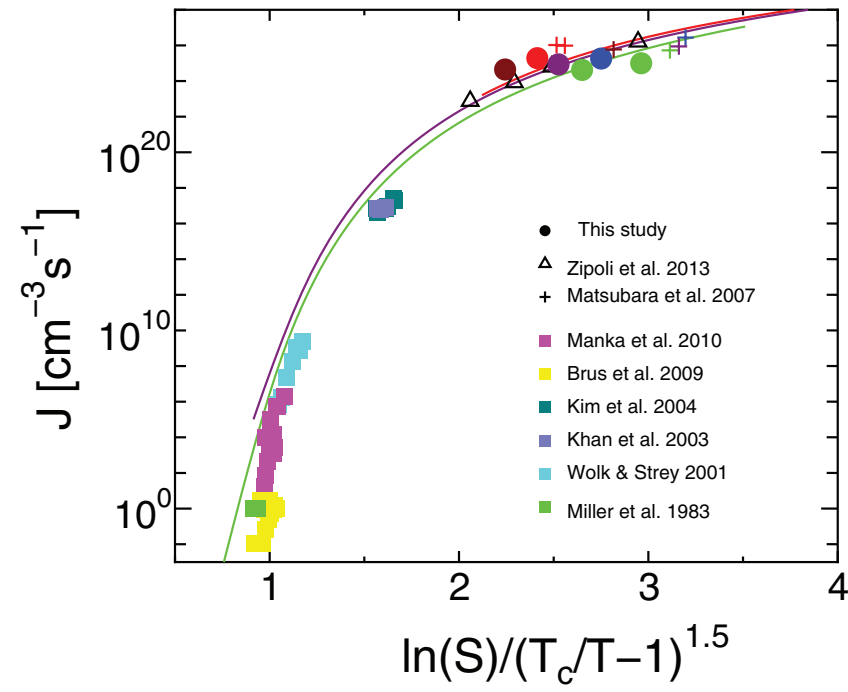

FIG. 5. The nucleation rates as a function of $\ln S /\left(T_{c} / T-1\right)^{1.5}$. The data by the MD simulations and the theoretical predictions by the SP model $(316 \mathrm{~K}$, $346 \mathrm{~K}$, and $383 \mathrm{~K}$ ) are the same as those in Fig. 3. For the MD simulations using SPC/E water model, we set $T_{c}=630 \mathrm{~K} \cdot{ }^{47}$ We also put the experimental data for nucleation of water by Miller et al. ${ }^{6}$ Wölk and Strey, ${ }^{20}$ Khan et al. ${ }^{22}$ Kim et al. ${ }^{23}$ Brus et al., ${ }^{26}$ and Manka et al. ${ }^{27}$ For the experimental results and Zipoli et al. ${ }^{57} T_{c}$ is set to be $647 \mathrm{~K}^{20}$

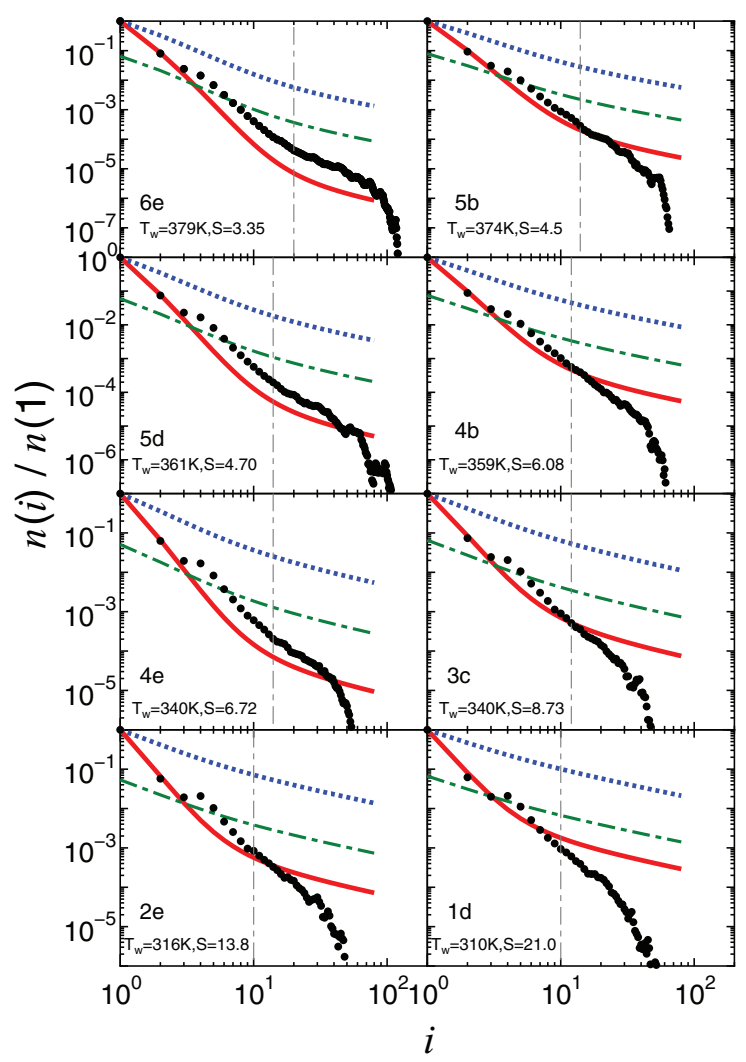

FIG. 6. The size distribution of clusters $n(i)$ obtained by the MD simulations is indicated by closed circles for the typical case. The size distributions obtained by the SP model (solid curve), the MCNT (dotted curve), and the CNT (dotted-dashed curve) are also plotted. The dotted-dashed lines show the size of critical clusters evaluated by the MD simulations. 


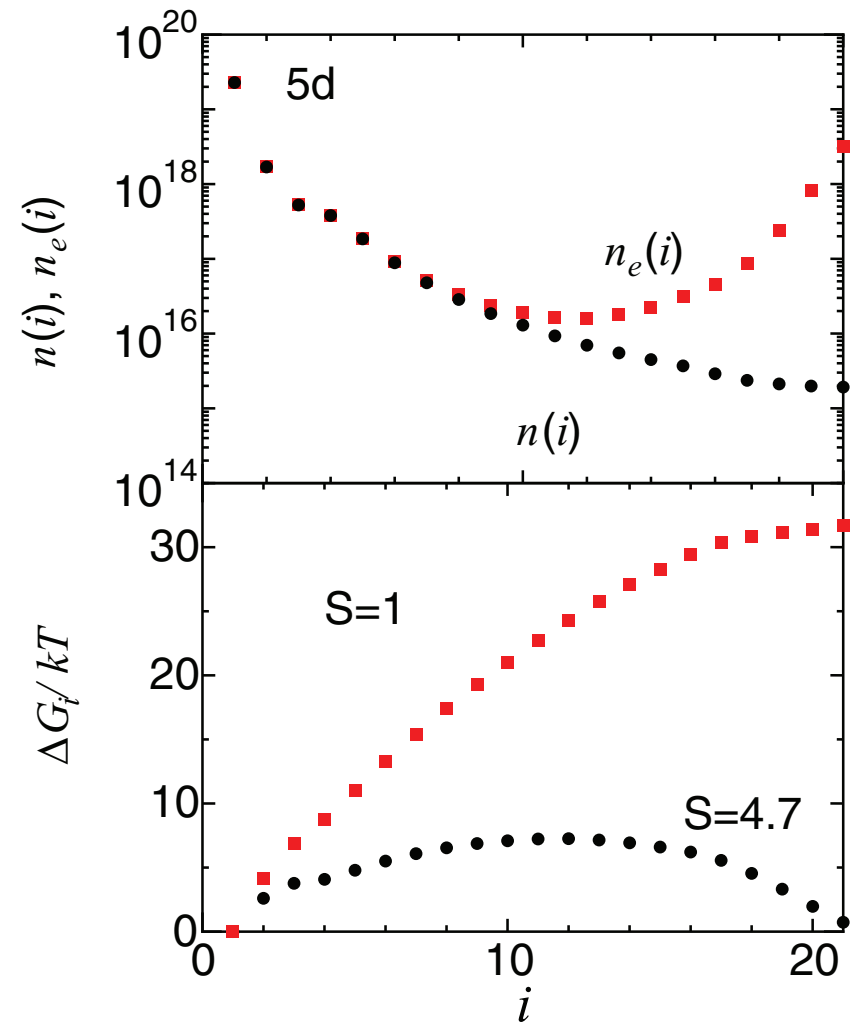

FIG. 7. The size distribution of clusters obtained by MD simulations (filled circles) and the equilibrium one (filled squares) in run $5 \mathrm{~d}$ are shown in the top panel. In the bottom panel, the formation free energies of a cluster $\Delta G_{i}(S$ $=1) / k T$ are shown for $S=4.7$ (filled circles) and $S=1$ (filled squares).

the theoretical models are plotted with solid lines (SP model) and dashed lines (MCNT). The SP model exhibits much better agreement with the numerical results than the MCNT. The SP model reproduces the formation free energy of a cluster to within $10 \%$. The CNT also reproduces the formation free

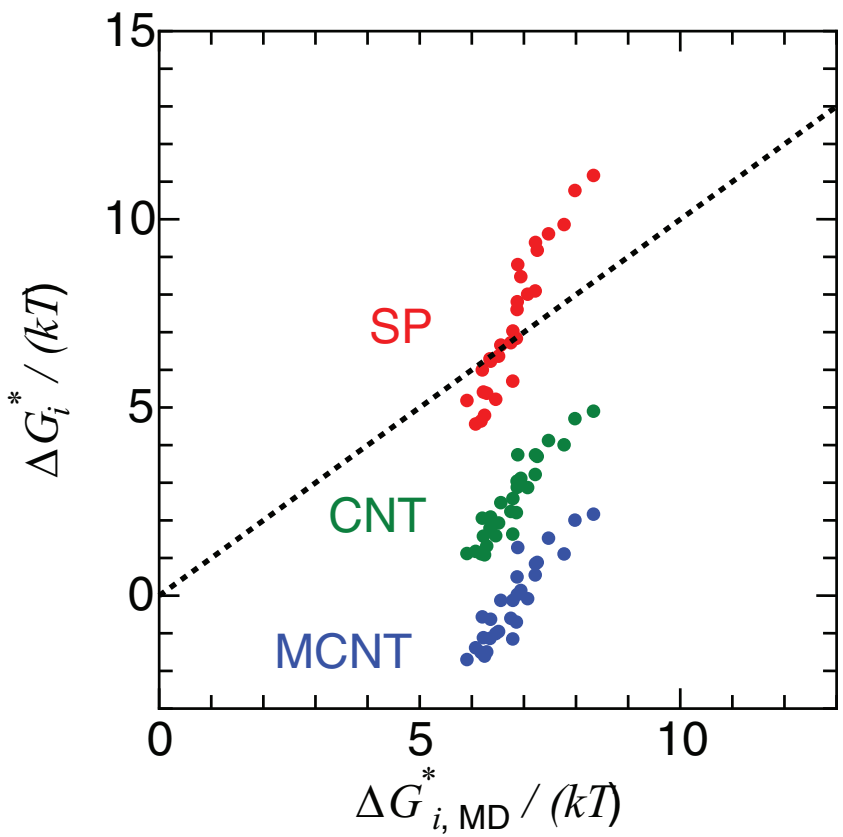

FIG. 8. The comparison of the maximum values of the formation free energy obtained by theoretical models and MD simulations $\Delta G_{\mathrm{i}, \mathrm{MD}}^{*}$.

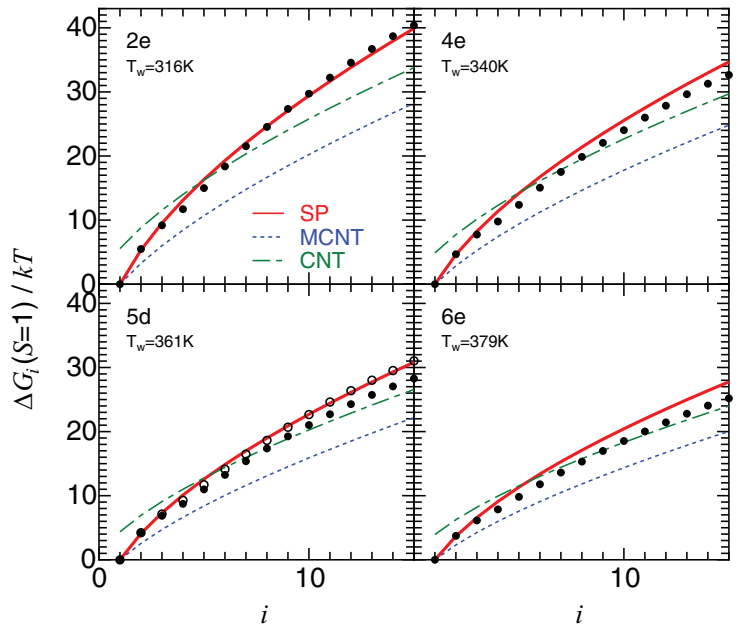

FIG. 9. The formation free energy of a cluster $\Delta G_{i}(S=1) / k T$ at various temperatures is shown by the filled circles. $T_{\mathrm{w}}=316 \mathrm{~K}$ (run $\left.2 \mathrm{e}\right), 340 \mathrm{~K}(4 \mathrm{e})$, $361 \mathrm{~K}(5 \mathrm{~d})$, and $379 \mathrm{~K}(6 \mathrm{e})$. The values obtained by the SP model (solid lines), the MCNT (dotted lines), and the CNT (dotted-dashed lines) are also shown. In run $5 \mathrm{~d}$, we also plotted the results of $T_{\mathrm{w}}=359 \mathrm{~K}$ (run $4 \mathrm{~b}$ ) with open circles.

energy of a cluster better than the MCNT, but the gradient of the CNT data is different to the numerical results.

From $\Delta G_{i}$ obtained by MD simulations, we can calculate the nucleation rate by using

$$
J=\left\{\sum_{i=1}^{\infty} \frac{1}{R^{+}(i) n_{\mathrm{e}}(i)}\right\}^{-1},
$$

where $n_{\mathrm{e}}(i)$ is given by Eq. (2). We confirmed the nucleation rates given by Eq. (10) predict $J_{\mathrm{MD}}$ measured by the MD simulations within the accuracy of $1 \%$ for all runs.

\section{E. Sticking probability}

As shown in Sec. II, the nucleation rate is obtained from the equilibrium number density $n_{e}(i)$ and the accretion rate which is proportional to the sticking probability $\alpha$. Using the growth rate of stable clusters obtained in the present MD simulation, we evaluated the sticking probability.

Tanaka et al. ${ }^{48}$ evaluated the sticking probability from the growth rate of stable clusters, $d i / d t$, neglecting the effect of evaporation. In the present study the evaporation effect was taken into account based on the method of Diemand et al. ${ }^{49}$ The growth rate of clusters, $d i / d t$, is given by

$$
\frac{d i}{d t}=R^{+}(i)-R^{-}(i)
$$

From Eqs. (4) and (5), we have

$$
\frac{d i}{d t}=4 \pi r_{0}^{2} i^{2 / 3} \alpha n(1) v_{\text {th }}\left[1-\left(\frac{i-1}{i}\right)^{2 / 3} e^{\frac{\Delta G_{i}-\Delta G_{i-1}}{k T}}\right] \text {, }
$$

where we use $n_{\mathrm{e}}(i)=n(1) \exp \left[-\Delta G_{i} /(k T)\right]$.

As shown in Eq. (8), $\Delta G_{i}$ has a bulk term $(\propto i)$ and a surface term $\left(\propto i^{2 / 3}\right)$. Since the other terms in Eq. (8) are smaller than these terms, the sum, $\Delta G_{i}+i k T \ln S$, is approximately 


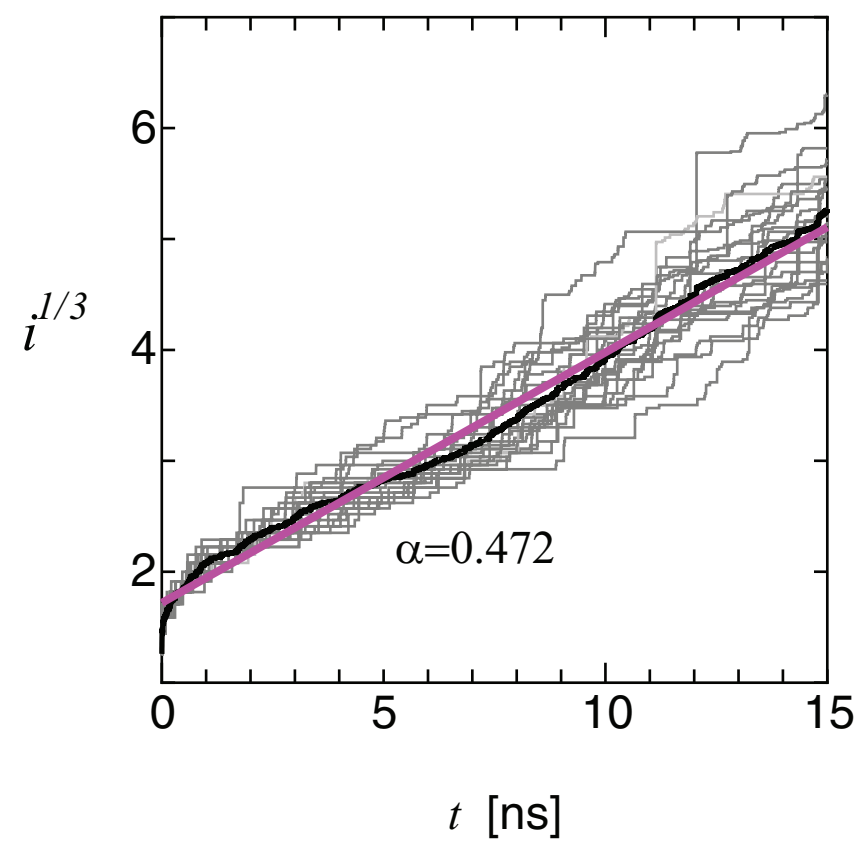

FIG. 10. Time evolution of the size of the largest cluster for 20 runs of $5 \mathrm{~d}$. The average value is plotted by a black line. Based on the averaged slope (red line), the sticking probability was found to be 0.472 .

proportional to $i^{2 / 3}$ and

$$
\Delta G_{i}-\Delta G_{i-1} \simeq-k T \ln S
$$

for $i \gg 1$. Hence, for $i \gg 1$, we obtain

$$
\frac{d i}{d t}=\alpha n(1) v_{\mathrm{th}}\left(4 \pi r_{0}^{2} i^{2 / 3}\right)\left[1-\frac{1}{S}\right] .
$$

Equation (14) suggests that the evaporation becomes considerable when the supersaturation ratio is small. Accordingly, the sticking probability is given by

$$
\alpha=\frac{3}{4 \pi r_{0}^{2} v_{\mathrm{th}} n(1)}\left(1-\frac{1}{S}\right)^{-1} \frac{d i^{1 / 3}}{d t} .
$$

The time derivative of $i^{1 / 3}$ was evaluated from the MD simulations. Figure 10 shows the time derivative of $i^{1 / 3}$ for run $5 \mathrm{~d}$. Based on the slope, the sticking probability was measured to be 0.472 . Using Eq. (15), we obtained the sticking probability for all runs. The values of $\alpha$ are also listed in Table II. The results are shown in Figure 11(a) as a function of supersaturation rate. By plotting a line of best fit, we obtained an empirical formula for the sticking probability:

$$
\ln \alpha=1.16 \ln S\left(\frac{T}{273 \mathrm{~K}}\right)^{3.3}-5.3
$$

(see Fig. 11(b)). The sticking probability increases with the supersaturation ratio, which is consistent with the LennardJones system. ${ }^{48,49}$ The formula (16) is valid within the parameter range of $S$ in which our MD simulations are done. In this range $(S>3)$, Eq. (16) indicates that $\alpha$ increases with the temperature for a fixed $S$. In the case of the flat surface and the equilibrium state $(S=1)$, on the other hand, the decreasing of $\alpha$ with the increasing temperature is reported by
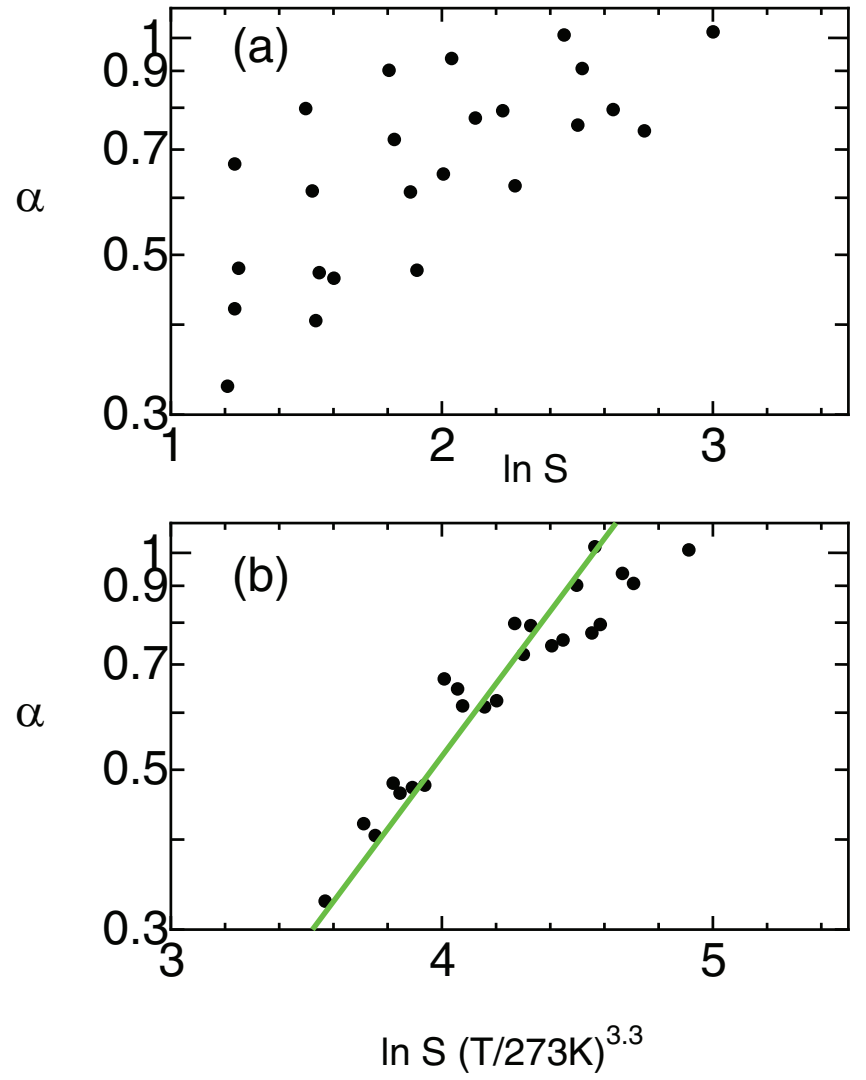

FIG. 11. (a) The sticking probabilities obtained by MD simulations as a function of supersaturation. (b) The sticking probabilities as a function of $\ln S(T / 273 \mathrm{~K})^{3.3}$. The equation of the line of best fit is $\ln \alpha$ $=1.16 \ln S(T / 273 \mathrm{~K})^{3.3}-5.3$, which is shown by a solid line.

the previous study. ${ }^{64}$ Around the equilibrium state $(S \simeq 1)$, the sticking probability should be examined in the future work.

We evaluated $\alpha$ by using the growth rate of clusters larger than the critical size with Eq. (15). Matsubara et al. ${ }^{47}$ evaluated the sticking probability (or the sticking coefficient) by directly measuring the condensation flux (i.e., accretion rate) for clusters smaller than or comparable to the critical size instead of the growth rate. Their values are one-order-of-magnitude greater than ours. This would indicate that the sticking probability might be different between the under-critical and the over-critical sizes. Matsubara et al. ${ }^{47}$ suggested that their large sticking coefficients are due to the large surface area of clusters. For small clusters, larger surface areas than the simple prediction with the bulk density are also reported by other studies. ${ }^{30,65}$ A high supersaturation ratio in Matsubara et al. ${ }^{47}$ can be another factor which causes the above difference in $\alpha$.

\section{SUMMARY AND FUTURE WORK}

Our results on the nucleation of water molecules are summarized as follows.

1. The cluster size distributions, the formation free energy of a cluster, and the nucleation rates obtained from the MD simulations are compared with the predictions by the theoretical models (see Figures 3-6 and Table II). The CNT and the MCNT overestimate the nucleation rates (or the number density of critical clusters) for all 
runs. On the other hand, the SP model gives a better prediction. The nucleation rates predicted by the SP model are within one order of magnitude. The free energy of cluster formation $\Delta G_{i}$ (or the cluster integrals) for small clusters $(i \lesssim 20)$ is also well predicted by the SP model (with $10 \%$ accuracy) for a wide range of temperature. In this study, only large supersaturation ratios and nucleation rates were considered. In order to verify the validity of the SP model for smaller supersaturation ratios, simulations using a larger number of molecules are necessary.

2. The sticking probability of vapor molecules onto clusters was measured in supersaturated conditions, by using the growth rate of stable clusters in the MD simulations. The sticking probability $\alpha$ increases with the supersaturation ratio. This tendency is the same as exhibited by the Lennard-Jones system. ${ }^{48,49}$ The lowest value of $\alpha$ was observed to be 0.3 for $T \simeq 380 \mathrm{~K}$ and $S \simeq 3.3$. It is expected that $\alpha$ would be much smaller than 0.3 for lower supersaturation ratios. For such low- $S$ cases, we must estimate $\alpha$ precisely for the evaluation of $J$.

\section{ACKNOWLEDGMENTS}

We are grateful to Dr. Matsubara for discussions and valuable comments. H. Tanaka and K. K. Tanaka acknowledge Dr. Diemand and Dr. Angelil for fruitful discussions. We would like to thank the anonymous reviewers for their valuable suggestions to improve the quality of the paper. This work was supported by the Japan Society for the Promotion of Science (JSPS).

\section{APPENDIX: RELATION BETWEEN EQ. (7) AND THE PREVIOUS FORMULA (REF. 47)}

In the present paper, we use Eq. (7) to obtain the equilibrium number density. In this Appendix, we show the relation, Eq. (7), to the formula used in Matsubara et al. ${ }^{47}$ Equation (7) is a recurrence relation and yields

$$
\begin{aligned}
\frac{n_{\mathrm{e}}(i)}{n(i)} & =\prod_{k=1}^{i-1}\left(1-\frac{J}{R^{+}(k) n(k)}\right)^{-1} \\
& =\prod_{k=1}^{i-1} \exp \left\{-\ln \left(1-\frac{J}{R^{+}(k) n(k)}\right)\right\} .
\end{aligned}
$$

If we assume

$$
\frac{J}{R^{+}(i) n(i)} \ll 1
$$

for all $i$, we obtain

$$
\begin{aligned}
\frac{n_{\mathrm{e}}(i)}{n(i)} & =\prod_{k=1}^{i-1} \exp \left\{-\frac{J}{R^{+}(k) n(k)}\right\} \\
& =\exp \left\{-J \sum_{k=1}^{k-1} \frac{1}{R^{+}(k) n(k)}\right\},
\end{aligned}
$$

which corresponds to the formula (27) in Matsubara et al. ${ }^{47}$ Since the nucleation rate is expressed with the Zeldovich fac- tor $Z$ :

$$
\begin{aligned}
& J \simeq R^{+}\left(i_{*}\right) n_{\mathrm{e}}\left(i_{*}\right) Z, \\
& Z=\left\{\frac{-1}{2 \pi k T}\left(\frac{d^{2} \Delta G_{i}}{d i^{2}}\right)_{i=i_{*}}\right\}^{1 / 2},
\end{aligned}
$$

the condition (A2) is written as

$$
Z \ll 1 .
$$

In the theory of CNT, MCNT, and SP model, the Zeldovich factor is given by

$$
Z=\frac{1}{3} i_{*}^{-2 / 3} \sqrt{\frac{\eta}{\pi}+\frac{\xi}{\pi} i_{*}^{-1 / 3}},
$$

where $Z$ in the CNT (or MCNT) corresponds to the case of $\xi=0$. Equation (A6) indicates that the condition (A2) is satisfied in the case of $i^{*} \gg(\eta / 9 \pi)^{3 / 4} \sim 1$.

${ }^{1}$ M. Volmer and A. Weber, Z. Phys. Chem. 119, 277 (1926).

${ }^{2}$ V. R. Becker and W. Döring, Ann. Physik. 416, 719 (1935).

${ }^{3}$ J. B. Zel'dovich, J. Exp. Theor. Phys. 12, 525 (1942).

${ }^{4}$ J. Feder, K. C. Russell, J. Lothe, and G. M. Pound, Adv. Phys. 15, 111 (1966).

${ }^{5}$ J. L. Schmitt, G. W. Adams, and R. A. Zalabsky, J. Chem. Phys. 77, 2089 (1982).

${ }^{6}$ R. C. Miller, R. J. Anderson, J. L. Kassner, Jr., and D. E. Hagen, J. Chem. Phys. 78, 3204 (1983).

${ }^{7}$ J. L. Schmitt, R. A. Zalabsky, and G. W. Adams, J. Chem. Phys. 79, 4496 (1983).

${ }^{8}$ G. W. Adams, J. L. Schmitt, and R. A. Zalabsky, J. Chem. Phys. 81, 5074 (1984).

${ }^{9}$ A. Dillmann and G. E. A. Meier, J. Chem. Phys. 94, 3872 (1991).

${ }^{10}$ D. W. Oxtoby, J. Phys. Condens. Matter 4, 7627 (1992).

${ }^{11}$ D. Wright, R. Caldwell, C. Moxely, and M. S. ElShall, J. Chem. Phys. 98, 3356 (1993).

${ }^{12}$ C. F. Delale and G. E. A. Meier, J. Chem. Phys. 98, 9850 (1993).

${ }^{13}$ Y. Viisanen, R. Strey, and H. Reiss, J. Chem. Phys. 99, 4680 (1993).

${ }^{14}$ A. Laaksonen, I. J. Ford, and M. Kulmala, Phys. Rev. E 49, 5517 (1994).

${ }^{15}$ Y. Viisanen and R. Strey, J. Chem. Phys. 101, 7835 (1994).

${ }^{16}$ D. Kane and M. S. El-Shall, J. Chem. Phys. 105, 7617 (1996).

${ }^{17}$ K. Hämeri and M. Kulmala, J. Chem. Phys. 105, 7696 (1996).

${ }^{18}$ C. C. M. Luijten, K. J. Bosschaart, and M. E. H. van Dongen, J. Chem. Phys. 106, 8116 (1997).

${ }^{19}$ M. P. Anisimov, P. K. Hopke, I. N. Shaimordanov, S. D. Shandakov, and L.-E. Magnusson, J. Chem. Phys. 115, 810 (2001).

${ }^{20}$ J. Wölk and R. Strey, J. Phys. Chem. B 105, 11683 (2001).

${ }^{21}$ V. B. Mikheev, P. M. Irving, N. S. Laulainen, S. E. Barlow, and V. V. Pervukhin, J. Chem. Phys. 116, 10772 (2002).

${ }^{22}$ A. Khan, C. H. Heath, U. M. Dieregsweiler, B. E. Wyslouzil, and R. Strey, J. Phys. Chem. B 119, 3138 (2003).

${ }^{23}$ Y. J. Kim, B. E. Wyslouzil, G. Wilemski, J. Wölk, and R. Strey, J. Phys. Chem. A 108, 4365 (2004).

${ }^{24}$ V. Holten, D. G. Labetski, and M. E. H. van Dongen, J. Chem. Phys. 123, 104505 (2005).

${ }^{25}$ D. Brus, V. Ždímal, and J. Smolík, J. Chem. Phys. 129, 174501 (2008).

${ }^{26}$ D. Brus, V. Ždímal, and H. Uchtmann, J. Phys. Chem. A 131, 074507 (2009).

${ }^{27}$ A. A. Manka, D. Brus, A.-P. Hyvärinen, H. Lihavainen, J. Wölk, and R. Strey, J. Chem. Phys. 132, 244505 (2010).

${ }^{28}$ I. J. Ford, Phys. Rev. E 56, 5615 (1997).

${ }^{29}$ I. Kusaka, A.-G. Wang, and J. H. Seinfeld, J. Chem. Phys. 108, 3416 (1998).

${ }^{30}$ K. Yasuoka and M. Matsumoto, J. Chem. Phys. 109, 8451 (1998).

${ }^{31} \mathrm{~K}$. Yasuoka and M. Matsumoto, J. Chem. Phys. 109, 8463 (1998).

${ }^{32}$ P. R. ten Wolde and D. Frenkel, J. Chem. Phys. 109, 9901 (1998).

${ }^{33}$ P. R. ten Wolde, M. J. Ruiz-Montero, and D. Frenkel, J. Chem. Phys. 110, 1591 (1999).

${ }^{34}$ K. J. Oh and X. C. Zeng, J. Chem. Phys. 110, 4471 (1999).

${ }^{35}$ I. Kusaka and D. W. Oxtoby, J. Chem. Phys. 110, 5249 (1999). 
${ }^{36}$ B. Senger, P. Schaaf, D. S. Corti, R. Bowles, D. Pointu, J.-C. Voegel, and H. Reiss, J. Chem. Phys. 110, 6438 (1999).

${ }^{37}$ K. J. Oh and X. C. Zeng, J. Chem. Phys. 112, 294 (2000).

${ }^{38} \mathrm{~S}$. Tanimura, K. Yasuoka, and T. Ebisuzaki, J. Chem. Phys. 112, 3812 (2000).

${ }^{39}$ H. Vehkamäki and I. J. Ford, J. Chem. Phys. 112, 4193 (2000).

${ }^{40}$ K. Laasonen, S. Wonczak, R. Strey, and A. Laaksonen, J. Chem. Phys. 113, 9741 (2000).

${ }^{41}$ P. Schaaf, B. Senger, J.-C. Voegel, R. K. Bowles, and H. Reiss, J. Chem. Phys. 114, 8091 (2001).

${ }^{42}$ S. Yoo, K. J. Oh, and X. C. Zeng, J. Chem. Phys. 115, 8518 (2001).

${ }^{43}$ S. Toxvaerd, J. Chem. Phys. 115, 8913 (2001).

${ }^{44}$ B. Chen, J. I. Siepmann, K. J. Oh, and M. L. Klein, J. Chem. Phys. 115, 10903 (2001).

${ }^{45}$ S. Toxvaerd, J. Chem. Phys. 119, 10764 (2003).

${ }^{46}$ K. K. Tanaka, H. Tanaka, K. Kawamura, and K. Nakazawa, J. Chem. Phys. 122, 184514 (2005).

${ }^{47}$ H. Matsubara, T. Koishi, T. Ebisuzaki, and K. Yasuoka, J. Chem. Phys. 127, 214507 (2007).

${ }^{48}$ K. K. Tanaka, H. Tanaka, T. Yamamoto, and K. Kawamura, J. Chem. Phys. 134, 204313 (2011).

${ }^{49}$ J. Diemand, R. Angelil, K. K. Tanaka, and H. Tanaka, J. Chem. Phys. 139, 074309 (2013).

${ }^{50}$ D. Reguera, R. K. Bowles, Y. Djikaev, and H. Reiss, J. Chem. Phys. 118, 340 (2003).
${ }^{51}$ D. Reguera and H. Reiss, Phys. Rev. Lett. 93, 165701 (2004).

${ }^{52}$ V. I. Kalikmanov, J. Chem. Phys. 124, 124505 (2006).

${ }^{53}$ J. Wedekind, J. Wölk, D. Reguera, and R. Strey, J. Chem. Phys. 127, 154515 (2007).

${ }^{54}$ J. Merikanto, E. Zapadinsky, A. Lauri, I. Napari, and H. Vehkamäki, J. Chem. Phys. 127, 104303 (2007).

${ }^{55}$ V. I. Kalikmanov, J. Wölk, and T. Kraska, J. Chem. Phys. 128, 124506 (2008).

${ }^{56}$ H. J. Berendsen, J. R. Grigera, and T. P. Straatsma, J. Chem. Phys. 91, 6269 (1987).

${ }^{57}$ F. Zipoli, T. Laino, S. Stolz, E. Martin, C. Winkelmann, and A. Curioni, J. Chem. Phys. 139, 094501 (2013).

${ }^{58}$ R. Schulz, B. Lindner, L. Petridis, and J. C. Smith, J. Chem. Theory Comput. 5, 2798 (2009).

${ }^{59}$ W. L. Jorgensen, D. S. Maxwell, and J. Tirado-Rives, J. Am. Chem. Soc. 118, 11225 (1996)

${ }^{60}$ K. K. Tanaka, J. Diemand, R. Angelil, and H. Tanaka, "Free energy of cluster formation and a new scaling relation for the nucleation rate," $\mathrm{J}$. Chem. Phys. (submitted).

${ }^{61}$ B. N. Hale, J. Chem. Phys. 122, 204509 (2005).

${ }^{62}$ J. Qian, E. Stöckelmann, and R. Hentschke, J. Mol. Model 5, 281 (1999).

${ }^{63} \mathrm{H}$. Matsubara, private communication (2013).

${ }^{64}$ M. Matsumoto, Fluid Phase Equilib. 125, 195 (1996).

${ }^{65}$ R. Angelil, J. Diemand, K. K. Tanaka, and H. Tanaka, J. Chem. Phys. 140, 074303 (2014). 